\title{
MiR-17-5p modulates osteoblastic differentiation and cell proliferation by targeting SMAD7 in non-traumatic osteonecrosis
}

\author{
Jie Jia ${ }^{1,3}$, Xiaobo Feng ${ }^{1,3}$, Weihua Xu ${ }^{1}$, Shuhua Yang ${ }^{1}$, Qing Zhang ${ }^{2}$, Xianzhe Liu ${ }^{1}$, Yong Feng ${ }^{1}$ \\ and Zhipeng Dai ${ }^{1}$
}

MicroRNAs (miRNAs) have recently been recognized to have a role in human orthopedic disorders. The objective of our study was to explore the expression profile and biological function of miRNA-17-5p (miR-17-5p), which is well known to be related to cancer cell proliferation and invasion, in osteoblastic differentiation and in cell proliferation. The expression levels of miR-17$5 p$ in the femoral head mesenchymal stem cells of 20 patients with non-traumatic osteonecrosis (ON) and 10 patients with osteoarthritis (OA) were examined by quantitative reverse transcription-PCR (qRT-PCR). Furthermore, the interaction between miR-17-5p and SMAD7 was observed. We found that in non-traumatic ON samples the level of mature miR-17-5p was significantly lower than that of OA samples $(P=0.0002)$. By targeting SMAD7, miR-17-5p promoted nuclear translocation of $\beta$-catenin, enhanced expression of COL1A1 and finally facilitated the proliferation and differentiation of HMSC-bm cells. We also demonstrated that restoring expression of SMAD7 in HMSC-bm cells partially reversed the function of miR-17-5p. Together, our data suggested a theory that dysfunction of a network containing miR-17-5p, SMAD7 and $\beta$-catenin could contribute to $\mathrm{ON}$ pathogenesis. The present study prompts the potential clinical value of miR-17-5p in non-traumatic $\mathrm{ON}$. Experimental \& Molecular Medicine (2014) 46, e107; doi:10.1038/emm.2014.43; published online 25 July 2014

\section{INTRODUCTION}

Osteonecrosis $(\mathrm{ON})$ of the femoral head is a common disease that regularly affects patients aged $30-50$ years and is characterized by destruction of the blood supply of the femoral head. ${ }^{1,2}$ The causes of non-traumatic ON are complex. ${ }^{3}$ One study indicated that altered osteogenic differentiation capability of mesenchymal stem cells (MSCs) is associated with non-traumatic $\mathrm{ON}$, and the differentiation potential of MSCs in patients with non-traumatic ON differs according to the risk factor. ${ }^{3}$ This finding implies one possible theory, that is, the decreased replication capacity of MSCs or decreased MSC activity in the bone marrow may have an important role in non-traumatic ON. ${ }^{4}$

MicroRNAs (miRNAs) are small, noncoding RNAs that potentially regulate thousands of genes. ${ }^{5-8}$ It is believed that miRNAs manipulate $30 \%$ of the human genome, primarily by binding to the $3^{\prime}$-untranslated region (UTR) of their target
mRNAs, resulting in translational repression or mRNA degradation. ${ }^{9-11}$ Although miRNAs are known to behave as regulators in many diseases, there have been few reports that expound on osteoblastic genesis-related miRNAs. ${ }^{7}, 12$ MiRNA17-5p (miR-17-5p) has been proven to regulate cell proliferation and differentiation in lung, breast and colorectal cancer cell lines. ${ }^{13-15}$ Therefore, miR-17-5p is one of the most representative miRNAs that can impact proliferation and differentiation. However, the relationships between miR-17-5p, MSCs and non-traumatic ON remain unclear.

In the present study, we showed a discrepancy in the expression levels of miR-17-5p in MSCs of non-traumatic ON patients and hip osteoarthritis (OA) patients. To reveal the potential etiology of non-traumatic $\mathrm{ON}$, we performed a functional study and identified that miR-17-5p could promote the osteogenesis of MSCs through directly targeting and

\footnotetext{
${ }^{1}$ Department of Orthopedics, Union Hospital, Tongji Medical College, Huazhong University of Science and Technology, Wuhan, China and ${ }^{2}$ Department of Blood Transfusion, Union Hospital, Tongji Medical College, Huazhong University of Science and Technology, Wuhan, China

${ }^{3}$ These authors contributed equally to this work.

Correspondence: Professor W Xu or Professor S Yang, Department of Orthopedics, Union Hospital, Tongji Medical College, Huazhong University of Science and Technology, Jiefang Avenue No.1277, Wuhan 430030, China.

E-mail: xwh1230@163.com or shuhua_yang@yeah.net

Received 5 December 2013; revised 19 April 2014; accepted 2 May 2014
} 
downregulating SMAD7. Furthermore, it has been reported that forced expression of SMAD7 could retard osteoblastic cell proliferation stimulated by TGF- $\beta 1,{ }^{16}$ suggesting that miR-17$5 \mathrm{p}$ might regulate osteoblastic cell proliferation in part by restraining SMAD7 expression. Therefore, miR-17-5p might be used as a potential biomarker and therapeutic target of non-traumatic $\mathrm{ON}$ in the future.

\section{MATERIALS AND METHODS}

\section{Subjects}

Upon approval from the ethics committee of Union Hospital and obtaining written informed consents, bone marrow samples were obtained from non-traumatic ON patients $(n=20)$ and OA patients $(n=10)$ undergoing surgery between April 2008 and July 2010 at Wuhan Union Hospital, Huazhong University of Science and Technology. Bone marrow samples $(5 \mathrm{ml})$ were obtained from the proximal end of the femurs while inserting a tapered awl into the femoral canal during total hip arthroplasty. Non-traumatic ON was diagnosed based on radiographic and magnetic resonance imaging. OA was confirmed using radiographic images, and the diagnostic criteria were satisfied for all patients. Of the 20 patients with non-traumatic ON, 11 patients were treated with corticosteroids, 7 patients suffered from alcoholism and 2 patients were diagnosed with idiopathic ON. The clinical characteristics of all patients are shown in Table 1.

Mononuclear cells from the bone marrow were separated by centrifugation on a Ficoll-Hypaque gradient (density $=1.077 \mathrm{~g} \mathrm{~cm}^{-3}$; Fresenius Kabi Norge, Lysker, Norway), suspended in low-glucose Dulbecco's modified Eagle's medium (HyClone, Logan, UT, USA) containing 10\% fetal bovine serum (FBS; Gibco, Carlsbad, CA, USA) and seeded at a density of $1 \times 10^{6}$ cells per $\mathrm{cm}^{2}$. After 10 days, the cultured cells were collected for reverse transcription-PCR.

\section{Cell culture and ALP staining}

HMSC-bm cells were purchased from the Cell Bank of the Type Culture Collection of the Chinese Academy of Sciences and suspended in MSC growth medium consisting of low-glucose Dulbecco's modified Eagle's medium supplemented with 10\% FBS. The cells were cultivated in culture flasks at a density of $1 \times 10^{5}$ cells per $\mathrm{cm}^{3}$ at $37^{\circ} \mathrm{C}$ in a humidified atmosphere containing $5 \% \mathrm{CO}_{2}$. Once reaching $90 \%$ confluency, the cells were passaged at 1:2 dilutions. Cells between the fourth and seventh passages were used for the experiments.

For cell-proliferation counting, cells were seeded into 96-well plates (2,500 perwell), and cell numbers were assessed using the Cell Counting Kit-8 (Dojindo, Kumamoto, Japan) every $24 \mathrm{~h}$ according to the manufacturer's instructions. The results were presented as the absorbance at $450 \mathrm{~nm}$.

Table 1 Characteristics of patients with osteoarthritis and osteonecrosis

\begin{tabular}{lccc}
\hline & No. of patients & Age (years) & Gender (F/M) \\
\hline $\begin{array}{l}\text { Osteoarthritis } \\
\text { Osteonecrosis }\end{array}$ & 10 & $53 \pm 7$ & $7 / 3$ \\
Alcohol & 7 & $52 \pm 14$ & $1 / 6$ \\
Steroid & 11 & $49 \pm 15$ & $5 / 6$ \\
Idiopathic & 2 & $52 \pm 8$ & $2 / 0$ \\
\hline
\end{tabular}

Abbreviations: $\mathrm{F}$, female; $\mathrm{M}$, male.
An ALP staining kit (Beyotime Institute of Biotechnology, Nanjing, Jiangsu, China) was used according to the procedures of the manufacturer and of a previous report. ${ }^{17}$

\section{Regulation of the miR-17-5p levels}

To alter miR-17-5p expression, cells were transiently transfected with has-miR-17-5p mimics (final concentration, $100 \mathrm{nM}$ ) or has-miR-17$5 p$ inhibitor (final concentration, $100 \mathrm{nM}$ ) using Lipofectamine 2000 (Invitrogen, Carlsbad, CA, USA). For osteoblastic differentiation, the medium was removed $6 \mathrm{~h}$ after transfection and substituted for fresh medium containing $10 \%$ FBS and $100 \mathrm{ng} \mathrm{ml}^{-1}$ BMP-2 (R\&D Systems, Minneapolis, MN, USA).

\section{Quantitative real-time PCR}

Total RNA of cells or tissues was extracted using the Trizol reagent (Invitrogen). qRT-PCR reactions were carried out with the Power SYBR Green PCR Master Mix and 7900HT thermocycler (Applied Biosystems, Carlsbad, CA, USA). Data were analyzed using the SDS Relative Quantification Software version 2.2 (Applied Biosystems), with the automatic threshold cycle (Ct) settings at baseline and the threshold for ${ }^{\Delta} \mathrm{Ct}$ determination; expression of the U6 small nucleolar RNA gene was used as an internal control. The results are presented as the comparative $\mathrm{Ct}$ for each miRNA. Each qRT-PCR was performed in triplicate. The $20-\mu \mathrm{l}$ qRT-PCR reaction contained the following reagents: $9 \mu \mathrm{l}$ SYBR Green Mix, $2 \mu \mathrm{l}$ miRNA reverse transcription (RT) product, $2 \mu$ Bulge-Loop miRNA Forward Primer $(5 \mu \mathrm{M}), 2 \mu \mathrm{l}$ BulgeLoop miRNA Reverse Primer ( $5 \mu \mathrm{M}$; RiboBio Co., Ltd, Guangzhou, Guangdong, China) and RNase-free $\mathrm{H}_{2} \mathrm{O}$ in a final volume of $20 \mu \mathrm{l}$. The reactions were incubated at $95^{\circ} \mathrm{C}$ for $20 \mathrm{~s}$, followed by 40 cycles of $95^{\circ} \mathrm{C}$ for $10 \mathrm{~s}, 60^{\circ} \mathrm{C}$ for $20 \mathrm{~s}$ and $70^{\circ} \mathrm{C}$ for $10 \mathrm{~s}$ and finally $95^{\circ} \mathrm{C}$ for $10 \mathrm{~s}$, $60{ }^{\circ} \mathrm{C}$ for $20 \mathrm{~s}$ and $95^{\circ} \mathrm{C}$ for $10 \mathrm{~s}$.

\section{miRNA target prediction and luciferase reporter assay} TargetScan version 6.2 (http://www.targetscan.org/vert_50) and Pictar (http://pictar.mdc-berlin.de/) were used to predict the miR-17-5p targets. Information on the complete computational protocols are available at the corresponding websites. The miR-17-5p target region of the SMAD7-3'UTR (sequence containing 5'-AAAUAAAGAAAAGAUGCACUUUG-3', part of the NCBI RefSeq ID of NM_005904) was inserted into the pmiR-RB-Report vector and named SMAD7$3^{\prime}$ UTR WT (RiboBio Co., Ltd). As a control, the plasmid (sequence $5^{\prime}$-AAAUAAAGAAAAGUACGUGAAAG- ${ }^{\prime}$ ) containing the mutant sequence of the miR-17-5p target region of the SMAD7-3'UTR was also inserted into the pmiR-RB-Report vector and named SMAD7$3^{\prime}$ UTR MT. Co-transfection of 200 ng SMAD7-3'UTR WT or SMAD7-3'UTR MT with $100 \mathrm{nM}$ of miR-17-5p mimic or miR17-5p inhibitor and their negative controls was carried out using jetPRIME (Polyplus-transfection Inc., New York, NY, USA) in 48-well plates, respectively. Each experiment was repeated four times.

\section{Preparation of cytoplasmic and nuclear fractions}

The NE-PER kit (Pierce, Waltham, MA, USA) was used to prepare cytoplasmic and nuclear fractions. ${ }^{18}$ Cell membranes were destroyed to deliver the cytoplasmic contents. Integrated nuclei were retrieved from the cytoplastic extracts by centrifugation, and the nuclei were washed with PBS and disrupted to harvest the nuclear extract. Blots were visualized using an Odyssey IR scanner (Li-Cor, Lincoln, NE, USA) with the Odyssey imaging software 3.0 (Li-Cor). 


\section{Western blotting}

Extracts of cell lysate and tissue were analyzed by western blotting using the IRDye $800 \mathrm{CW}$-conjugated antibody and LI-COR imaging system (LI-COR Biosciences, Lincoln, NE, USA) according to the manufacturer's instructions. Membranes were scanned and analyzed using an Odyssey IR scanner and Odyssey imaging software 3.0.

\section{Osteoblastic differentiation}

Osteoblastic differentiation was achieved by administering media containing $10 \%$ FBS and $100 \mathrm{ng} \mathrm{ml}^{-1}$ BMP-2 (R\&D Systems).

\section{Statistics}

Experimental data were formulated as the means \pm s.d. of triplicate independent samples. As most of the variables were small, nonparametric tests were performed as the statistical method. Statistical significance for two groups was analyzed using the Mann-Whitney test. All other data were analyzed using Kruskal-Wallis analysis of variance, and the Dunn's multiple comparison test was applied as a posttest. To assess the correlation between the miR-17-5p and SMAD7 expression levels, the Pearson correlation coefficient was used. Statistical analyses were performed with SPSS 13.0 (SPSS Inc., Chicago, IL, USA) for Windows (Microsoft Corp., Seattle, WA, USA), and statistical significance was defined as $P<0.05$.

\section{RESULTS}

Differential expression of miR-17-5p between MSCs of nontraumatic $\mathrm{ON}$ patients and $\mathrm{OA}$ patients

To study the expression of miR-17-5p in non-traumatic ON, we first evaluated the expression of miR-17-5p in all MSC samples from non-traumatic $\mathrm{ON}$ patients and $\mathrm{OA}$ patients using quantitative PCR (Figure 1a). The expression levels of mature miR-17-5p were lower in the MSCs from the nontraumatic $\mathrm{ON}$ group compared with the OA group. All samples were categorized by etiology, and we found a significant difference in the level of miR-17-5p between the two groups $(P=0.0002)$.

The elevated expression of $\mathrm{miR}-17-5 \mathrm{p}$ is indispensable for BMP-2-induced osteoblastic differentiation

To determine the potential roles of miR-17-5p during proliferation and osteoblastic differentiation of MSCs, the expression pattern of miR-17-5p was analyzed in the stromal cell line HMSC-bm, which was derived from bone marrow. The results are summarized in Figure 1b. miR-17-5p expression was decreased after a temporary rise in the number of cells that proliferated spontaneously, but had no osteoblastic-inductive BMP-2, over 7 days, which indicated that miR-17-5p may positively regulate cell proliferation during MSC differentiation.

When HMSC-bm cells were treated with BMP-2, the drop in the level of miR-17-5p receded compared with that in cells with no additional osteoblastic-inductive material. We observed that the BMP-2-treated HMSC-bm cells maintained growth, even after long treatment duration. Taken together, these findings suggest that, upon BMP-2 treatment, cell proliferation is maintained by increasing the effect of miR17-5p, that miR-17-5p may positively influence osteoblastic a
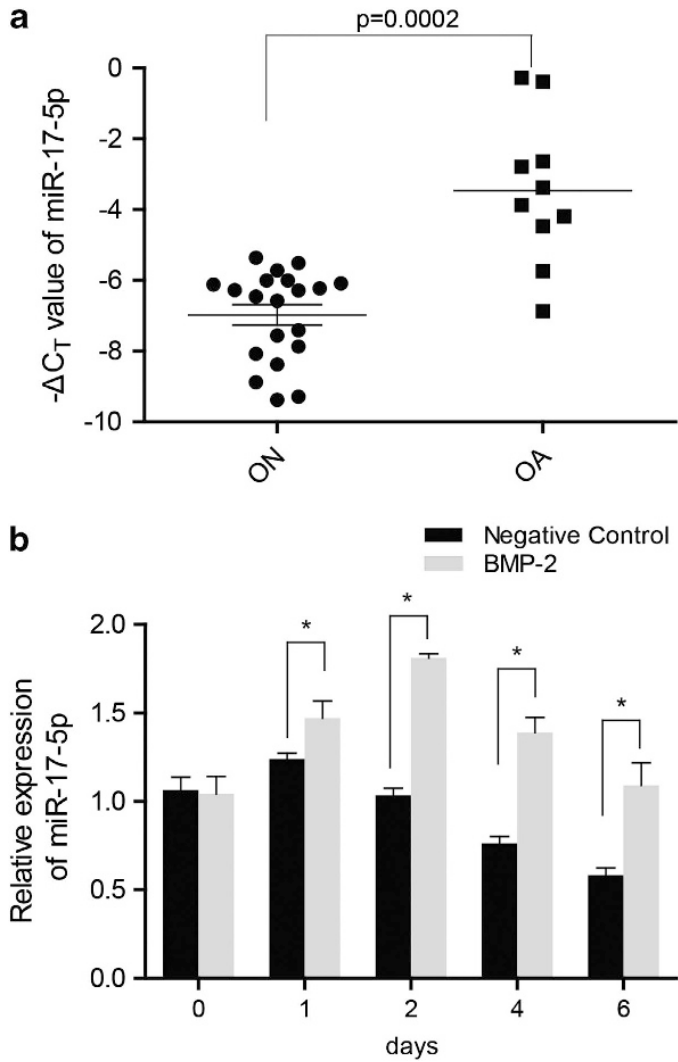

Figure 1 Differential expression of microRNA-17-5p (miR-17-5p) in patients and HMSC-bm cell lines. (a) Quantitative reverse transcription-PCR analysis of the expression of mature miR-17-5p in the primary culture cells obtained from $O A$ and non-traumatic ON patients. The expression of mature miR-17-5p is significantly higher in cells from osteonecrosis (non-traumatic ON) compared with those from hip osteoarthritis $(O A)$ patients $(P=0.0002)$. (b) MiR-17-5p expression in HMSC-bm cells stimulated by BMP-2. Cells were harvested at the indicated times, and the endogenous expression level of miR-17-5p was measured. Solid and white bars indicate samples treated with and without BMP-2, respectively. $* P<0.05$.

differentiation and that miR-17-5p should be low during osteoblastic differentiation and proliferation of MSCs.

SMAD7 is a target and is negatively regulated by miR-17-5p To explore the mechanisms by which miR-17-5p effects proliferation and osteoblastic differentiation of MSCs, we chose the miRNA target prediction databases Pictar and TargetScan to determine the possible target genes of miR-175 p. Based on results using both databases, SMAD7, which is involved in BMP-2-induced osteoblastic differentiation and contains a conserved $3^{\prime} \mathrm{UTR}$ element, was selected for further study (Figure 2a).

To determine whether miR-17-5p could repress SMAD7, we created $3^{\prime}$ UTR reporter plasmids containing the wild-type $3^{\prime} \mathrm{UTR}$ (SMAD7-3'UTR-WT) or a mutant with the predicted miR-17-5p 3'UTR sites (SMAD7-3'UTR MT) of SMAD7. Luciferase analysis suggested that miR-17-5p could 


\title{
a 3' gauggacgugacaUUCGUGAAAc 5' hsa-miR-17 | | || | || | \\ 1351:5' aaauaaagaaaagAUGCACUUUg 3' SMAD7
}

\author{
3' gauggacgugacaUUCGUGAAAc 5' mmu-miR-17 \\ | | | || || | \\ 1399:5' gaauaaagaaaagAUGCACUUUg 3' Smad7
}
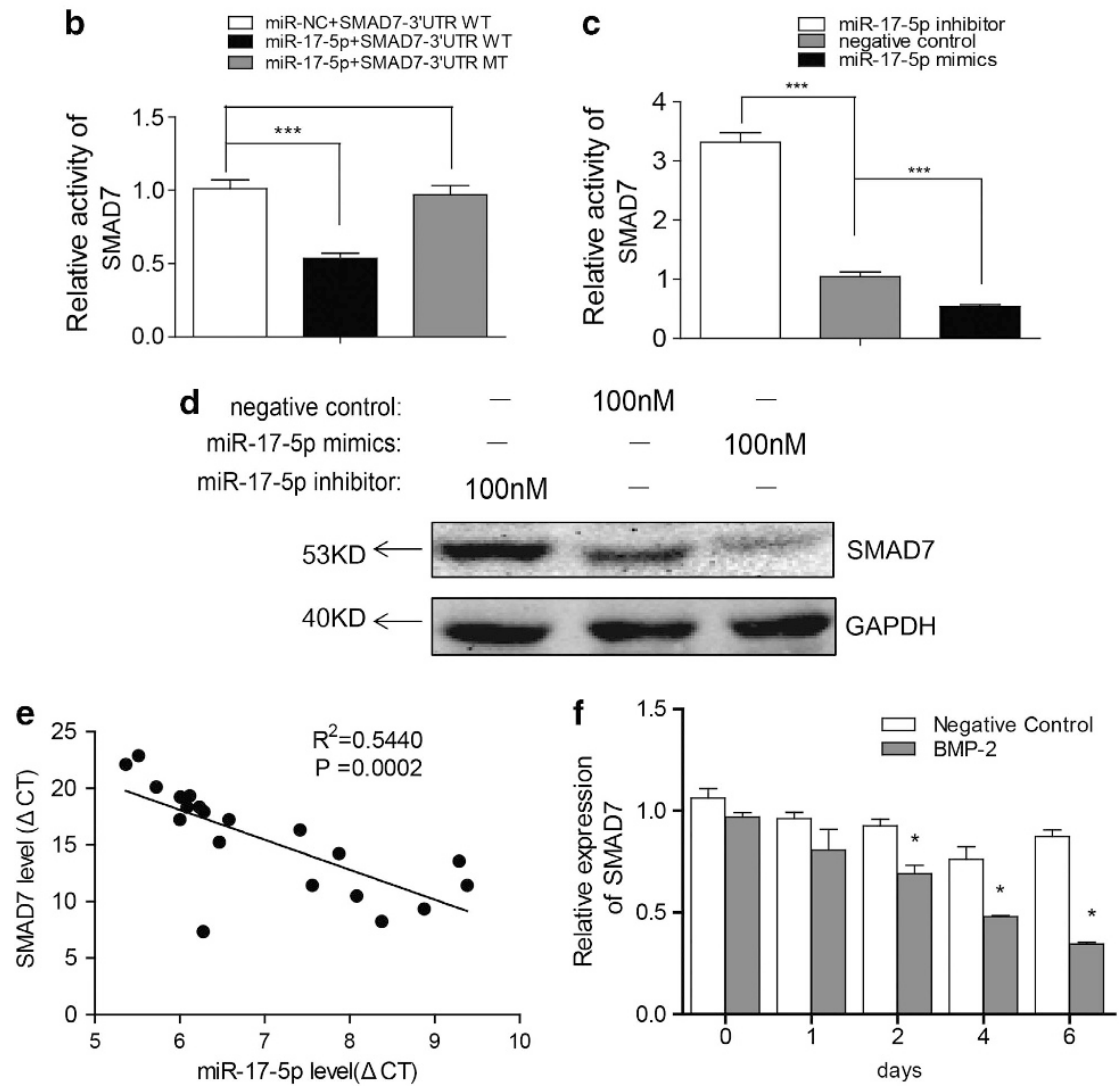

Figure 2 SMAD7 is a target and is negatively regulated by microRNA-17-5p (miR-17-5p). (a) Schematic diagram of the putative miR-17$5 p$ seed sequences in the $3^{\prime}$ - untranslated regions (UTRs) of the Smad7 mRNAs of Homo sapiens and Mus musculus. Hsa indicates Homo sapiens, and Mmu indicates Mus musculus. (b) Relative activity of luciferase reporters with a wild-type or mutant Smad7-3'UTR after cotransfection with miR-17-5p mimics or NC mimics in HMSC-bm cells, ${ }^{* *} P<0.001$. (c) Relative activity of luciferase reporters with a wild-type or mutant Smad7-3'UTR after cotransfection with miR-17-5p inhibitor or NC inhibitor in HMSC-bm cells, *** $P<0.001$. (d) Protein levels of Smad7 in miR-17-5p-transfected or miR-17-5p inhibitor-treated HMSC-bm cells. (e) Correlation analysis for the expression of SMAD7 and miR-17-5p in the osteoarthritis specimens mentioned in Figure 1a. (f) SMAD7 expression in HMSC-bm cells stimulated by BMP-2. Cells were harvested at the indicated times, and the expression level of SMAD was measured. Solid and white bars indicate samples treated with and without BMP-2, respectively. ${ }^{*} P<0.05$ between the negative-control and BMP-2 groups. GAPDH, glyceraldehyde 3-phosphate dehydrogenase.

downregulate the luciferase activity of the SMAD7-3'UTR-WT plasmid, but not that of SMAD7-3'UTR-MT in comparison with the negative-control miRNAs (Figure 2b). Figure $2 \mathrm{c}$ demonstrates that the mRNA level of SMAD7 was markedly decreased or elevated after the administration of miR-17-5p mimics or miR-17-5p inhibitor, respectively, in HMSC-bm cells compared with control cells, and the protein level of SMAD7 displayed the same trend as that of the mRNA (Figure 2d). As expected, the negative correlation between miR-17-5p and SMAD7 in ON tissues of 20 patients was proven (Figure $2 \mathrm{e}, R^{2}=0.5440, P<0.001$ ). Furthermore, it has been reported that SMAD7 might act as a suppressor of bone formation, ${ }^{19}$ so we examined the enrichment of SMAD7 during osteoblast differentiation. Interestingly, SMAD7 expression was steadily downregulated during bone formation after administration of BMP-2, which was the opposite of the expression pattern of miR-17-5p (Figure 2f).

\section{MiR-17-5p has a key role in HMSC-bm osteoblastic} differentiation and growth

To investigate the effect of miR-17-5p on osteoblastic differentiation of HMSC-bm cells, we altered the miR-17-5p level in the cells. After transfection of miR-17-5p mimics or 

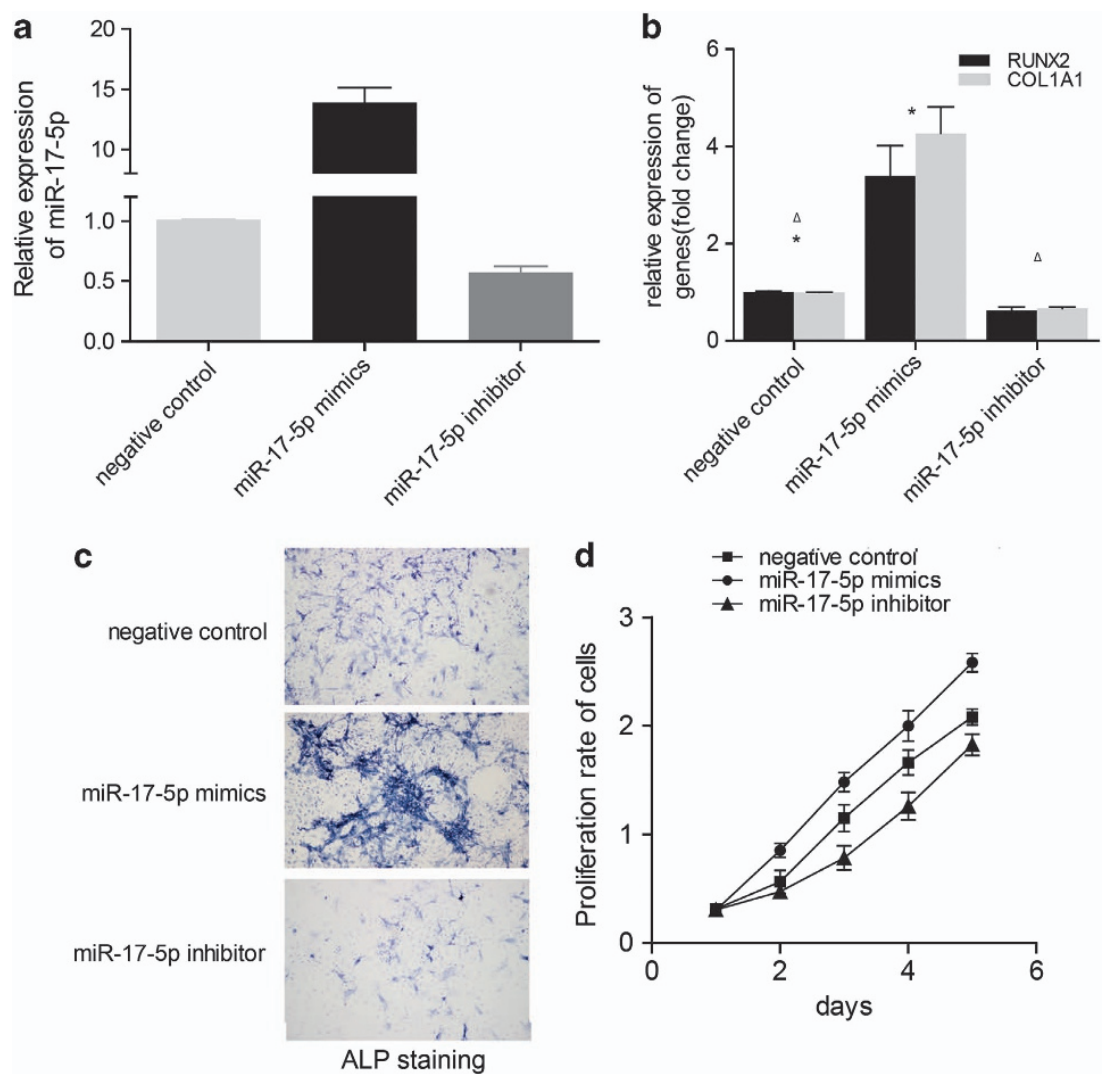

Figure 3 Biological influence of transfection of microRNA-17-5p (miR-17-5p) mimics or miR-17-5p inhibitor on osteoblastic differentiation and proliferation. (a) Relative level of miR-17-5p in HMSC-bm cells $48 \mathrm{~h}$ after transfection with miR-17-5p mimics (100 nM), miR-17-5p inhibitor (100 nM) or negative control. The miR-17-5p mimics or miR-17-5p inhibitor were transfected, and BMP-4 was added after $4 \mathrm{~h}$. After 6 days, RUNX2 and COL1A1 expression levels were measured (b), and ALP staining (c) was performed. The relative proliferation of cells was examined (d). ${ }^{*} P<0.05 . \Delta$ indicates $P<0.05$.

miR-17-5p inhibitor, qRT-PCR analysis showed that the level of miR-17-5p was increased by the miR-17-5p mimics and decreased by the miR-17-5p inhibitor (Figure 3a), which showed that the cellular level of miR-17-5p in this system could be regulated by transfecting these reagents. Then, HMSC-bm cells were transfected with miR-17-5p mimics or miR-17-5p inhibitor accompanied by the induction of osteoblastic differentiation through the addition of BMP-2. The ALP activity and expression level of the RUNX2, COL1A1 and osteocalcin transcripts were measured to determine bone formation. As expected, compared with transfection of the negative control, the expression of RUNX2 and COL1A1 was increased by transfection of miR-17-5p mimics (Figure 3b). ALP staining was also promoted by transfection of miR-17-5p mimics (Figure 3c). Conversely, the expression of RUNX2 and ALP staining were both retarded by transfection of the miR17-5p inhibitor (Figures 3b and c). Yet, late-stage osteoblastic markers, such as the absence of osteocalcin gene expression (the Ct value by qRT-PCR was greater than 35), were not observed in the cultures. Furthermore, CCK8 experiments showed that miR-17-5p mimics could boost, whereas miR-17$5 p$ inhibitor impeded, the proliferation of HMSC-bm cells (Figure 3d). In conclusion, our data demonstrated that miR- 17-5p has a potential role in facilitating osteoblastic differentiation and proliferation in cells in vitro.

Negative modulation of SMAD7 expression by miR-17-5 is crucial for miR-17-5p-promoted HMSC-bm cell proliferation and bone formation

Because miR-17-5p could repress the expression of SMAD7, we wondered if SMAD7 is responsible for the miR-17-5ppromoted HMSC-bm cell proliferation and osteoblastic differentiation. Therefore, we synthesized a SMAD7 expression plasmid using the pcDNA3.1-myc (-) vector (named pcDNA3.1-SMAD7) to study its mechanisms. Figure 4 illustrates that SMAD7 could negatively regulate proliferation and the markers of bone formation; moreover, restoration of SMAD7 blocked the majority of miR-17-5p-induced proliferation (Figures 4a and c) and ALP staining (Figure 4b). In addition, qRT-PCR showed that SMAD7 reversed the upregulation of RUNX2 and COL1A1 that was generated by miR17-5p (Figure 4c). Taken together, these data suggested that SMAD7 can depress HMSC-bm cell proliferation and bone formation ability, which is indispensable for the pro-osteoblastic differentiation capability of miR-17-5p exposed to BMP-2 stimuli. 
a

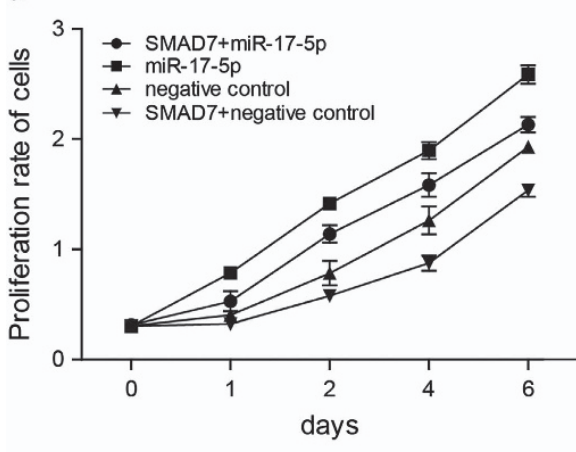

b

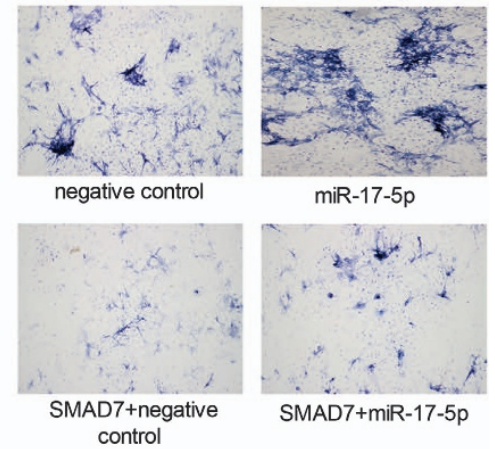

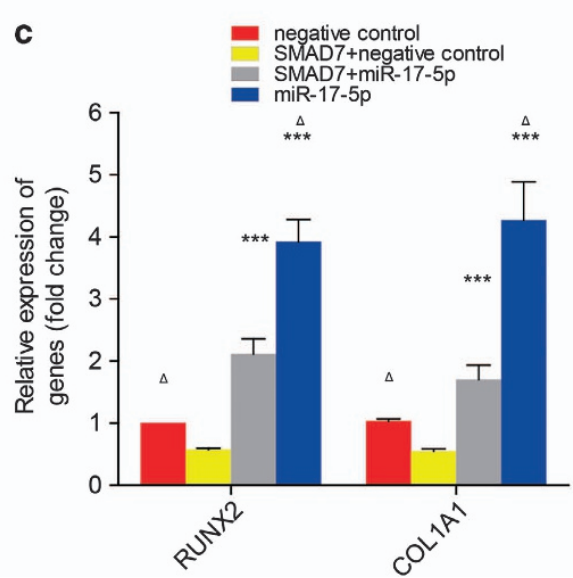

Figure 4 Negative modulation of SMAD7 expression by microRNA-17-5p (miR-17-5p) is crucial for MiR-17-5p-promoted HMSC-bm cell proliferation and bone formation. Negative modulation of Smad7 expression by miR-17-5p is crucial for miR-17-5p-promoted HMSC-bm cell proliferation and bone formation. MiR-17-5p and control mimics were co-transfected with or without pcDNA3.1-SMAD7 (SMAD7), and BMP-2 was added after $4 \mathrm{~h}$. After 6 days, the relative proliferation rate of the cells was examined (a), and ALP staining (b) and measurement of RUNX2 and COL1A1 expression (c) were performed. ${ }^{* *} P<0.001 . \Delta$ indicates $P<0.05$.

The reduction of SMAD7 upon miR-17-5p exposure results in nuclear translocation and transactivation of $\beta$-catenin signaling

It has been reported that BMP-2 promotes bone formation by stabilizing $\beta$-catenin and increases the transcription of nuclear $\beta$-catenin. ${ }^{20-22}$ We wondered whether miR-17-5p facilitates the nuclear translocation of $\beta$-catenin by inhibiting the activity of SMAD7. After co-transfecting a $\beta$-catenin reporter gene with miR-17-5p mimics or inhibitor, we observed that the activity of $\beta$-catenin signaling was increased or decreased, respectively (Figure 5a). Meanwhile, restoration of SMAD7 expression effectively eliminated the miR-17-5p-enhanced, $\beta$-catenin reporter gene activity (Figure 5a). Furthermore, transfection of miR-17-5p mimics or inhibitor only was sufficient to enhance or reduce the amount of nuclear $\beta$-catenin (Figure 5b, left panel). Similar to the reporter gene assay, overexpression of SMAD7 could abrogate the miR-17-5ppromoted accumulation of $\beta$-catenin in the nucleus (Figure 5b, right panel). Taken together, our data suggested that the cytoplasmic-nuclear translocation of $\beta$-catenin is responsible for the osteoblastic differentiation and proliferation of HMSC-bm cells that are regulated by miR-17-5p/ SMAD7-signaling.

\section{DISCUSSION}

As one of the most common diseases observed by orthopedic surgeons, $\mathrm{ON}$ is a disorder that is difficult to diagnose because of the lack of early clinical symptoms. As with many other diseases with high morbidity and disability, vigilance and prediction can reveal the patients suffering from the illness but lacking severe symptoms. Most ON results from direct trauma, but non-traumatic ON should be suspected in patients with predilection for the disease. ON may result from direct trauma and non-traumatic causes, such as inflammation and other additive stresses on the anatomic and vascular integrity of the involved region.

In the present study, we reported that miR-17-5p, which is located in the miR-17/92 cluster and has important roles in various cancers, regulated osteoblastic differentiation and cell proliferation through directly targeting SMAD7. We identified miR-17-5p as a potential biomarker by measuring its diverse expression in non-traumatic ON patients and $\mathrm{OA}$ patients, but it should be emphasized that further studies with larger prospective cohorts should be conducted to verify the sensitivity and specificity of this miRNA biomarker. MiR-17-5p has been reported to facilitate the cell proliferation of different human cancer cells by inhibiting 


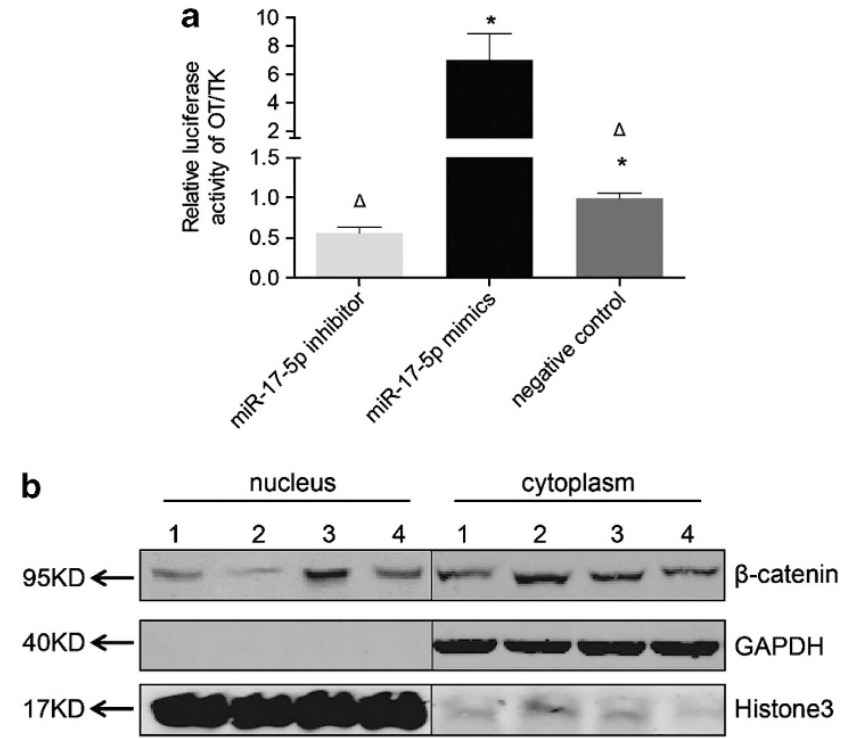

Figure 5 The reduction of SMAD7 by microRNA-17-5p (miR-17$5 p$ ) results in nuclear translocation and transactivation of $\beta$-catenin signaling. (a) Luciferase activity of a reporter construct harboring the $\beta$-catenin promoter after HMSC-bm cells were cotransfected with negative control, miR-17-5p mimics or miR-17-5p inhibitor for $72 \mathrm{~h} .{ }^{*} P<0.05$. $\Delta$ indicates $\mathrm{P}<0.05$. (b) HMSC-bm cells were transfected with the indicated substances for $72 \mathrm{~h}$. Then, the nuclear and cytoplasmic lysates from the total cell lysates were separated and subjected to western blot analysis (1: negative control; 2: negative control + SMAD7; 3: miR-20a mimics; 4: miR-17-5p+SMAD7). GAPDH, glyceraldehyde 3phosphate dehydrogenase.

important cancer-relevant genes, such as P21, BIM, AIB and E2F1, ${ }^{14,23,24}$ Similarly, our present study indicated that miR$17-5 \mathrm{p}$ boosted cell proliferation and osteoblastic differentiation in human MSCs, that is, HMSC-bm. Generally, miRNAs function as post-transcriptional repressors that exert their biological functions by suppressing the mRNA and protein expression of target genes. Our data showed that miR-17-5p downregulated the mRNA as well as protein levels of SMAD7 during these biological events and that transfection of SMAD7 partially retarded miR-17-5p-induced osteoblastic differentiation (Figure 4). SMAD7 has been proven as a key factor in embryonic development and adult homoeostasis, and abnormal expression of SMAD7 is often associated with human diseases, such as inflammatory diseases and tumors. Nevertheless, the role of miRNA-Smad7 regulation in nontraumatic $\mathrm{ON}$ is unclear.

Here, we demonstrated that decreased expression of miR$17-5 p$ is correlated with the worse clinical characteristics and poor survival rate of non-traumatic ON patients. Conversely, the ectopic expression of miR-17-5p could definitely provoke non-traumatic $\mathrm{ON}$ differentiation and proliferation in vitro via enhancing the nuclear translocation of $\beta$-catenin, which, for the first time, strongly indicates a pivotal role of the miR-17$5 \mathrm{p} / \mathrm{SMAD} 7 / \beta$-catenin pathway in the progression of nontraumatic ON.
The cytokine BMP-2, which activates a pathway that includes $\beta$-catenin protein phosphorylation and nuclear translocation, ${ }^{20,22,25,26}$ is highly expressed and stimulates osteoblast differentiation. In our present studies, we observed that the increased level of BMP-2 in HMSC-bm cells is responsible for the induction of miR-17-5p, which may further reinforce $\beta$-catenin-induced cell proliferation and osteoblastic differentiation, thus forming a positivefeedback loop.

In summary, BMP-2-induced expression of miR-17-5p has an important role in non-traumatic ON via directly repressing the transcription of SMAD7 and enhancing the activity of the $\beta$-catenin signaling pathway, which sheds light on the understanding of miRNA biology for resolving bone diseases such as non-traumatic ON. Illustrating the functions of miRNAs in osteoblast regulation in vivo could further help to find novel mechanisms of bone metabolism and differentiation.

\section{CONFLICT OF INTEREST}

The authors declare no conflict of interest.

\section{ACKNOWLEDGEMENTS}

This study was supported by the National Natural Science Foundation of China (No.81271969 and No.81101375).

1 Ficat RP. Idiopathic bone necrosis of the femoral head. Early diagnosis and treatment. J Bone Joint Surg Br 1985; 67: 3-9.

2 Mont MA, Marulanda GA, Jones LC, Saleh KJ, Gordon N, Hungerford DS et al. Systematic analysis of classification systems for osteonecrosis of the femoral head. J Bone Joint Surg Am 2006; 88(Suppl 3), $16-26$.

3 Lee JS, Roh HL, Kim CH, Jung JS, Suh KT. Alterations in the differentiation ability of mesenchymal stem cells in patients with nontraumatic osteonecrosis of the femoral head: comparative analysis according to the risk factor. J Orthop Res 2006; 24: 604-609.

4 Yan Z, Hang D, Guo C, Chen Z. Fate of mesenchymal stem cells transplanted to osteonecrosis of femoral head. J Orthop Res 2009; 27: 442-446.

5 Ahmed FE, Ahmed NC, Vos PW, Bonnerup C, Atkins JN, Casey M et al. Diagnostic microRNA markers to screen for sporadic human colon cancer in stool: I. Proof of principle. Cancer Genomics Proteomics 2013; 10: $93-113$.

6 van Wijnen AJ, van de Peppel J, van Leeuwen JP, Lian JB, Stein GS, Westendorf $\mathrm{JJ}$ et al. MicroRNA functions in osteogenesis and dysfunctions in osteoporosis. Curr Osteoporos. Rep 2013; 11: 72-82.

7 Yamasaki K, Nakasa T, Miyaki S, Yamasaki T, Yasunaga Y, Ochi M. Angiogenic microRNA-210 is present in cells surrounding osteonecrosis. J Orthop Res 2012; 30: 1263-1270.

8 Ukai T, Sato M, Akutsu H, Umezawa A, Mochida J. MicroRNA-199a-3p, microRNA-193b, and microRNA-320c are correlated to aging and regulate human cartilage metabolism. J Orthop Res 2012; 30: 1915-1922.

9 Lagos-Quintana M, Rauhut R, Lendeckel W, Tuschl T. Identification of novel genes coding for small expressed RNAs. Science 2001; 294: 853-858.

10 Lau NC, Lim LP, Weinstein EG, Bartel DP. An abundant class of tiny RNAs with probable regulatory roles in Caenorhabditis elegans. Science 2001; 294: 858-862.

11 Lee RC, Ambros V. An extensive class of small RNAs in Caenorhabditis elegans. Science 2001; 294: 862-864.

12 Duan Z, Choy E, Nielsen GP, Rosenberg A, lafrate J, Yang C et al. Differential expression of microRNA (miRNA) in chordoma reveals a role for miRNA-1 in Met expression. J Orthop Res 2010; 28: 746-752. 
13 Matsubara H, Takeuchi T, Nishikawa E, Yanagisawa K, Hayashita Y, Ebi H et al. Apoptosis induction by antisense oligonucleotides against miR-17$5 \mathrm{p}$ and miR-20a in lung cancers overexpressing miR-17-92. Oncogene 2007; 26: 6099-6105.

14 Hossain A, Kuo MT, Saunders GF. Mir-17-5p regulates breast cancer cell proliferation by inhibiting translation of AIB1 mRNA. Mol Cell Biol 2006; 26: 8191-8201.

15 Volinia S, Calin GA, Liu CG, Ambs S, Cimmino A, Petrocca F et al. A microRNA expression signature of human solid tumors defines cancer gene targets. Proc Natl Acad Sci USA 2006; 103: 2257-2261.

16 Nishimori S, Tanaka Y, Chiba T, Fujii M, Imamura T, Miyazono K et al. Smad-mediated transcription is required for transforming growth factorbeta 1-induced p57(Kip2) proteolysis in osteoblastic cells. J Biol Chem 2001; 276: 10700-10705.

17 Jiang S, Zu Y, Fu Y, Zhang Y, Efferth T. Activation of the mitochondriadriven pathway of apoptosis in human PC-3 prostate cancer cells by a novel hydrophilic paclitaxel derivative, 7-xylosyl-10-deacetylpaclitaxel. Int J Oncol 2008; 33: 103-111.

18 Mukai T, Otsuka F, Otani H, Yamashita M, Takasugi K, Inagaki K et al TNF-alpha inhibits BMP-induced osteoblast differentiation through activating SAPK/JNK signaling. Biochem Biophys Res Commun 2007 356: 1004-1010.

19 Yano M, Inoue Y, Tobimatsu T, Hendy G, Canaff L, Sugimoto T et al. Smad7 inhibits differentiation and mineralization of mouse osteoblastic cells. Endocr J 2012; 59: 653-662.

20 Zhang M, Yan Y, Lim YB, Tang D, Xie R, Chen A et al. BMP-2 modulates beta-catenin signaling through stimulation of Lrp5 expression and inhibition of beta-TrCP expression in osteoblasts. J Cell Biochem 2009; 108: 896-905

21 Zhang N, Wei P, Gong A, Chiu WT, Lee HT, Colman H et al. FoxM1 promotes beta-catenin nuclear localization and controls Wnt target-gene expression and glioma tumorigenesis. Cancer Cell 2011; 20: 427-442.

22 Lee JH, Kim BG, Ahn JM, Park HJ, Park SK, Yoo JS et al. Role of PI3K on the regulation of BMP2-induced beta-Catenin activation in human bone marrow stem cells. Bone 2010; 46: 1522-1532.

23 Fontana L, Fiori ME, Albini S, Cifaldi L, Giovinazzi S, Forloni M et al. Antagomir-17-5p abolishes the growth of therapy-resistant neuroblastoma through p21 and BIM. PLOS ONE 2008; 3: e2236.

24 O'Donnell KA, Wentzel EA, Zeller KI, Dang CV, Mendell JT. c-Mycregulated microRNAs modulate E2F1 expression. Nature 2005; 435 839-843.

25 Attisano L, Wrana JL. Signal transduction by the TGF-beta superfamily Science 2002; 296: 1646-1647.

26 Day TF, Guo X, Garrett-Beal L, Yang Y. Wnt/beta-catenin signaling in mesenchymal progenitors controls osteoblast and chondrocyte differentiation during vertebrate skeletogenesis. Dev. Cell 2005; 8: 739-750

(1) $(9)$ This work is licensed under a Creative Commons Attribution-NonCommercial-NoDerivs 3.0 Unported License. The images or other third party material in this article are included in the article's Creative Commons license, unless indicated otherwise in the credit line; if the material is not included under the Creative Commons license, users will need to obtain permission from the license holder to reproduce the material. To view a copy of this license, visit http://creativecommons.org/licenses/by-nc-nd/3.0/ 